\title{
Thyroid Dysfunction and Arterial Stiffness. Does the Restoration of Thyroid Function Tests Offer any Benefit?
}

\author{
Panagiotis Anagnostis ${ }^{1,2}$, Spyridon N. Karras ${ }^{2}$, Efthimios Gotsis ${ }^{1}$ and Ioanna Gouni- Berthold ${ }^{3, *}$
}

${ }^{1} 2^{n d}$ Propedeutic Department of Internal Medicine, Aristotle University of Thessaloniki, Hippokration Hospital, Thessa-
loniki, Greece; ${ }^{2}$ Unit of Reproductive Endocrinology, First Department of Obstetrics and Gynecology, "Papageorgiou'"
General Hospital, Medical School, Aristotle University of Thessaloniki, Thessaloniki, Greece; ${ }^{3}$ Center for Endocrinol-
ogy, Diabetes and Preventive Medicine, University of Cologne, Kerpener St. 62, 50937 Cologne, Germany

\begin{abstract}
A growing body of evidence suggests that hypothyroidism and hyperthyroidism, either as overt or subclinical states, are associated with increased risk for cardiovascular disease (CVD). Except for the increased prevalence of lipid disorders, coagulation abnormalities, endothelial dysfunction, cardiac arrhythmias, ventricular hypertrophy and hypertension have been proposed as the most plausible pathways for this association, increased arterial stiffness (AS) may also play a role. Increased AS has emerged recently as an early indicator of CVD in various studies. On the other hand, thyroid dysfunction, mainly hypothyroidism (overt and subclinical) seems to be associated with increased AS, although data are heterogeneous, emerging from different populations, with various methodologies of AS assessment and with variable periods of observation. Restoration of thyroid function with L-thyroxin replacement seems to reverse AS parameters, whereas data on the effect of normalization of thyroid function in hyperthyroidism on AS are inconsistent. The present review provides insights on the association between AS and thyroid dysfunction and discusses the available data about potential benefits of the restoration of thyroid function on AS.
\end{abstract}

Keywords: Arterial stiffness, thyroid dysfunction, hypothyroidism, hyperthyroidism subclinical hypothyroidism, subclinical hyperthyroidism.

\section{INTRODUCTION}

Thyroid dysfunction either as hypo- or hyperthyroidism seems to exert detrimental effects on the cardiovascular system, since many studies associate them with increased cardiovascular morbidity and mortality [1]. Similar risks have been reported for subclinical hypothyroidism (Sub Hypo) and hyperthyroidism (Sub Hyper) either endogenous or exogenous, especially in patients $<65$ years old [2-4], although the evidence for such an association between thyroid dysfunction and increased risk for cardiovascular events and/or mortality is not strong enough for some categories, such as the elderly ( $>70$ years of age) [5]. Even higher thyroidstimulating hormone (TSH) levels with the normal range may be associated with increased cardiovascular disease (CVD) risk, since an increase of $1 \mathrm{mIU} / 1$ in TSH levels was found to be associated with a $33 \%$ higher risk (HR 1.33; 95\% CI 1.03-1.73) for the occurrence of myocardial infarction in patients with manifest vascular diseases, after adjustment for possible confounders [6].

Many mechanisms for this interplay may be involved. In particular, hypothyroidism causes specific alterations in cardiovascular hemodynamics including decreased heart rate, impaired left ventricular systolic and diastolic function, increased systemic vascular resistance (SVR), cardiac

*Address correspondence to this author at the Center for Endocrinology, Diabetes and Preventive Medicine, University of Cologne, Cologne, Germany. Kerpener St. 62, 50937, Cologne, Germany; Tel: +49 221 478-4070; Fax:+49 221 478-4179; Email: ioanna.berthold@uni-koeln.de contractility and increased risk for atherosclerosis [7]. The latter is mediated through variable mechanisms, including hypertension (mainly diastolic), atherogenic lipid profile [(mainly increased levels of low-density-lipoprotein cholesterol (LDL-C)], hyperhomocysteinaemia, impaired flowmediated endothelium-dependent vasodilatation, increased levels of C-reactive protein (CRP) and coagulation abnormalities $[3,7,8]$. Hyperthyroidism also affects the cardiovascular system via many mechanisms, such as increased heart rate, risk of arrhythmias, mainly atrial fibrillation, [9], systolic hypertension [3, 8] activation of the reninangiotensin-aldosterone system (RAS) and up-regulation of left ventricular, aortic, and liver angiotensin II receptors [10], as well as increased carotid intima media thickness (c-IMT), a surrogate marker of atherosclerosis [11]. Under physiological conditions, thyroid hormones affect the heart and vascular system through diverse genomic and non-genomic pathways. In particular, triiodothyronine $\left(\mathrm{T}_{3}\right)$, the active thyroid hormone, binds to specific nuclear receptors in cardiomyocytes and vascular smooth cells (VSMC) stimulating the transcription of genes encoding structural and regulatory proteins (genomic actions). It also interferes with ion channels or pumps of the cellular membrane (non-genomic actions, including stimulation of the sarcolemmal sodium channel, inward-rectifying and voltage-activated potassium channels, as well as calcium pumps $[12,13] . T_{3}$ induces local vasodilatation via tissue thermogenesis which, in turn, results in decreased SVR and decreased diastolic blood pressure (DBP). Decreased DBP results in decreased afterload and increased preload through activation of the RAS. Except 
for this indirect mechanism, $\mathrm{T}_{3}$ exerts direct cardiac chronotropic and inotropic effects which lead to increased cardiac output [7].

Arterial stiffness (AS) is a term used to express the reduced elastic responsiveness of the vessel wall to the ventricular primary pressure wave [14]. The speed of this wave that moves away from the heart after the ventricular ejection can be measured and is called pulse wave velocity (PWV). AS is determined mainly by the elastin-to-collagen ratio in the vessel wall. Ageing process and CVD risk factors, such as hypertension, smoking, dyslipidaemia and diabetes mellitus, lead to increased AS due to elastic fibre degeneration, increased collagen and calcium concentration which result in dilation and hypertrophy mainly of the large arteries and the aorta [14]. PWV is the gold standard measurement of AS and is inversely associated with vascular compliance. PWV increases as the artery becomes stiffer, since it conducts the pulse wave faster than a more distensible vessel. PWV can be calculated by measuring the time needed for the arterial waveform to pass between 2 sites along a vascular segment or by getting separate recordings on a fixed point in the cardiac cycle. This is accomplished either by applanation tonometry or by echocardiography with pulse wave Doppler [15]. PWV is the most well-validated method, with high accuracy, reproducibility and correlation with CVD ourcomes [14-16]. $\mathrm{AS}$, measured by PWV either in the aorta or peripheral arteries has recently emerged as surrogate marker of atherosclerosis and independent predictor of CVD events [17-19]. A recent meta-analysis showed that an increase in aortic PWV by $1 \mathrm{~m} / \mathrm{s}$ corresponds to an adjusted risk increase of $14 \%$, $15 \%$, and $15 \%$ in total CVD events, CVD mortality and allcause mortality, respectively, with its predictive ability being higher in patients with a higher baseline CVD risk [19].

The purpose of the present review is to provide current data regarding a potential association between AS and thyroid dysfunction and to report on the available evidence regarding benefits of the normalization of thyroid function on AS.

\section{HYPOTHYROIDISM AND ARTERIAL STIFFNESS}

Except for the aforementioned association with atherosclerosis and CVD risk factors, hypothyroidism has also been related to increased AS in various studies, in different populations, with AS determined at different sites of the arterial wall. Increased AS may represent an early sign of the arteriosclerotic process before clinically apparent vascular disease $[14,15]$.

The first evidence for alterations in arterial function and structure in hypothyroid states appeared 16 years ago, when a rather quite small study (11 subjects with hypothyroidism, 15 euthyroid controls) showed that hypothyroid patients presented higher diastolic diameter, compliance, distensibility and wall thickness in radial artery than controls, although without any differences in carotid compliance. It must be stated that this study used A-mode ultrasonic device for measuring radial artery diameter and wall-thickness and $\mathrm{B}-\mathrm{M}$ mode echo-tracking device for carotid artery evaluation [20].

In a small study in the Greek population, including 65 subjects ( 59 females, mean age $44.4 \pm 13.5$ years), TSH negatively correlated with reflection time index (RTI), which is the time to the beginning of the reflected wave expressed as a percentage of cardiac period and is an estimate of PWV. TSH was also positively associated with central systolic BP (SBP), central pulse pressure and augmentation pressure, all markers of AS. PWV and AS were higher in hypothyroid subjects compared with euthyroid ones. Even mild increases in TSH within the normal range were associated with mild but statistically significant changes of AS (indicated by mild decreases in RTI) [21]. The latter observation was also confirmed by another study from the same group, including euthyroid postmenopausal women, which demonstrated a linear correlation between mean PWV values and TSH levels. Those with $\mathrm{TSH}>2.5 \mathrm{mIU} / 1$ had significantly higher PWV values compared with those $<2.5 \mathrm{mIU} / 1$. In multivariate analysis TSH and insulin resistance were the only predictors of PWV [22].

Furthermore, an older study of a smaller sample size than the previous one $(\mathrm{N}=24)$, but with the similar age and gender distribution, showed that patients with untreated overt hypothyroidism (TSH $>10 \mathrm{mIU} / \mathrm{l}$ ) presented increased AS compared with euthyroid individuals, as indicated by the lower RTI values and the significantly higher augmentation gradient (AG) of central arterial pressure- the difference between the first and second systolic peaks of the central pressure waveforms- and the significantly higher central augmentation index $(\mathrm{AI})$, that is the $\mathrm{AG}$ expressed as a percentage of the pulse pressure. Both studies assessed AS by recording peripheral pulse pressure waveforms using applanation tonometry at the radial artery [23].

Increased AS assessed by measurements at the common carotid artery has also been reported in another recent cohort of hypothyroid subjects, in comparison with euthyroid ones [24]. Importantly, in this study AS was positively associated with CRP levels (which were also higher in hypothyroid patients than in controls), an index of systematic inflammation, known to be elevated in hypothyroidism [7], thus providing, yet another pathway to explain the role of hypothyroidism in increased CVD risk [24]. This association remained significant after adjusting for traditional CVD risk factors, such as BP, serum lipids and body mass index (BMI) [24].

It has been shown that renal impairment leads to alterations in thyroid hormone metabolism, mainly SubHypo and low $\mathrm{T}_{3}$ levels [25]. On the other hand, it is well-known that end-stage renal disease (ESRD) patients are at increased CVD risk and increased AS is one of the main contributors to this risk [26]. Altered thyroid metabolism may in part play a role to this contribution. Indeed, in a study in ESRD patients, free- $\mathrm{T}_{3}$ levels were inversely associated with surrogates of AS, such as the AI, which was calculated from pulse waves of the radial artery by applanation tonometry, and carotid-femoral PWV (c-f PWV), measured by sequential recordings of the arterial pressure wave at the carotid and femoral arteries [27]. These associations were evident only in non-diabetics and remained significant after multiple regression analysis [27]. The inverse association between free $\mathrm{T}_{3}$ and AI was also confirmed by others and was again maintained after adjustment for potential confounders such as age, gender, diabetes and CVD [28]. The decrease in free $\mathrm{T}_{3}$ levels, may be due to the non-thyroidal illness syndrome 
(NTIS), a common entity in ESRD patients, which has also been associated with endothelial dysfunction [29] and systematic inflammation [30]. In a recent study, low free $\mathrm{T}_{3}$ levels were associated with increased AS in ESRD patients and NTIS, as well as with coronary artery calcification scores and mortality [31]. The inverse association between free $\mathrm{T}_{3}$ and $\mathrm{AI}$ corrected for heart rate, was also demonstrated in patients with the resistance to thyroid hormone (RTH) syndrome, a rare autosomal characterized by general or partial resistance of tissues to thyroid hormomes, clinical euthyroidism, but elevated free $\mathrm{T}_{4}$ and $\mathrm{T}_{3}$ levels and a nonsuppressed or elevated TSH level [32]. This study also showed an inverse association between free $\mathrm{T}_{4}$ levels and AI in controls [32].

Although the exact mechanisms underlying the association of hypothyroidism with AS are not fully understood, it can be speculated that increased AS in hypothyroidism is a consequence of endothelial dysfunction (due to dyslipidaemia, hyperhomocysteinaemia and increased CRP levels), which is the earliest stage of atherosclerosis and may lead to decreased arterial distensibility. Moreover, a direct effect of $\mathrm{TSH}$ on the vessel wall cannot be excluded [33].

\section{HYPERTHYROIDISM AND ARTERIAL STIFF- NESS}

Thyrotoxicosis seems to impair vascular elastic properties, via different pathways, as mentioned above. It has been associated with increased AS in various studies, either through the direct effects of thyroid hormones on the VSMC or by increase in pulse pressure and the development of a hypervolaemic state or indirectly through RAS activation [10-13]. More specifically, a study in patients with Graves' disease (GD) assessing systemic AS by aortic AI and by using applanation tonometry, found that compared with euthyroid subjects, hyperthyroid patients displayed increased AS and decreased subendocardial perfusion (mainly due to increased heart rate), which were positively associated with increased NT-pro-B-type natriuretic peptide (NT-pro-BNP), a marker of volume overload. These findings may further provide insights to the increased CVD risk in GD patients. However, it must be stated that this study lacked a control group [34]. The positive association of increased NT-pro$\mathrm{BNP}$, as well as asymmetric dimethylarginine (a marker of endothelial dysfunction), with free thyroid hormone levels and heart rate has also been confirmed by others [35]. Another study has been conducted in patients with GD and overt hyperthyroidism in which AS was estimated as pulse pressure (that is systolic-diastolic BP)/stroke volume, by using echocardiographic methodology. The study showed that total AS was higher in hyperthyroid patients than in euthyroid controls. Moreover, it was associated with a normal mean BP, indicating that the increase in total AS was not due to a higher arterial load but to the faster heart rate and presented significant improvement after beta-blockade [36]. Nonetheless, the association of overt thyrotoxicosis with increased AS was not confirmed by all studies. Indeed, untreated thyrotoxic patients showed decreased central AS (lower AI and AG) compared with healthy controls. These parameters of central AS were assessed by noninvasive recordings of peripheral pressure waveforms in radial artery by the principle of pulse wave analysis [37].
Another small cross-sectional study, including hospitalized patients with untreated GD and euthyroid subjects assessed AS by ultrasonographic methodolgy. The authors did not find any difference in AS parameters between hyperthyroid and euthyroid subjects, although there were strong associations between all AS parameters and plasma thyroid hormone concentrations in the GD patients [38]. However, another study reported increased AS in carotid common artery in hyperthyroid GD compared with euthyroid ones, although it lacked a control group [39].

In addition, important findings also have emerged from studies with euthyroid patients, but with previous hyperthyroidism. A more recent study that did use a control group assessed AS in euthyroid GD patients with orbitopathy compared with healthy individuals. The study showed that AS index was markedly increased and aortic strain and distensibility were significantly reduced in the GD group compared with controls. Weak correlations were found between the severity of orbitopathy and aortic distensibility as well as the AS indexes [40]. Apart from the predisposition of the aorta to the detrimental effects of hyperthyroidism on the vessel wall, other possible explanations for these findings may be the effect of autoimmune processes on the aorta, the increased levels of inflammatory cytokines in patients with GD that could contribute to the impaired aortic elastic properties, gene polymorphisms that may predispose both to GD and AS and the extrathyroidal expression of TSH-receptors that may be increased in patients with GD, as well as smoking, which affects both arterial distensibility and Graves' opthalmopathy [40].

Except for endogenous thyrotoxicosis, exogenous one may also affect AS, although there is only limited amount of data available. An animal study, in which exogenous thyrotoxicosis (by administering L-thyroxine for 8 weeks) was induced in Wistar rats showed that thyrotoxic animals demonstrated stiffer descending aortas compared with euthyroid ones only at the upper physiologic levels of stress or strain. More specifically, the aorta of thyrotoxic animals showed a significant increase in the internal and external diameters, the media area, the number of smooth muscle cell nuclei and the collagen density, as well as a decrease in the elastin laminae thickness and elastin density. These data may indicate a detrimental effect of exogenous thyrotoxicosis on the mechanical properties of the thoracic aorta and its hemodynamic homeostasis, leading to increased risk for vascular complications [41]. A study in humans evaluating the effects of longterm TSH suppressive therapy on cardiac function has also shown detrimental effects on left ventricular mass index, isovolumetric relaxation time and diastolic function compared to healthy controls not receiving thyroxine, although the authors did not assess AS parameters [42].

\section{SUBCLINICAL THYROID FUNCTION AND AR- TERIAL STIFFNESS}

\subsection{Subclinical Hypothyroidism (SubHypo)}

As mentioned above, there is evidence that SubHypo (a condition of elevated TSH with normal thyroid hormones) is associated with increased CVD-related morbidity and mortality, mainly due to its association with lipid abnormalities, coagulation disorders, cardiac dysfunction, diastolic hyper- 
tension and endothelial dysfunction, as in overt hypothyroidism [3]. Although data are insufficient compared with those available for overt hypothyroidism, increased AS may also exist in SubHypo states. In a well-designed study (one of the largest about this topic), carotid AS (parameter $\beta$ index) was assessed by echo-tracking technology in 93 patients with SubHypo and 90 euthyroid controls, with both groups comparable in terms of traditional atherosclerotic factors. SubHypo patients had higher AS $\beta$ indexes compared with euthyroid ones, independently of high-sensitivity-CRP (hsCRP) levels. Furthermore, this index was significantly positively correlated with age, smoking, SBP or DBP, TSH and hsCRP and negatively associated with pulse rate [43].

The correlation of SubHypo to increased AS was also shown by other studies of smaller sample size than the previous one, with $\mathrm{AS}$ assessed by applanation tonometry in radial artery [21, 44]. Increased brachial-ankle PWV (baPWV), another parameter of AS and a good independent predictor of coronary artery disease [45], was also found in SubHypo patients. Notably, DBP was also higher and correlated well with baPWV, indicating that its increase might be one of the main mechanisms responsible for increased AS in these patients [46].

\subsection{Subclinical Hyperthyroidism (SubHyper)}

To the best of our knowledge, no study exists evaluating the association of SubHyper with AS parameters, although either endogenous or exogenous subclinical thyrotoxicosis has been associated with various effects on the cardiovascular system, such as impaired exercise tolerance, atrial and ventricular arrhythmias, reduced heart rate variability, left ventricular hypertrophy, increased c-IMT and elevated plasma fibrinogen. However, additional studies are needed to determine if these cardiovascular risk factors are indeed responsible for the association of SubHyper with increased CVD risk and if they are reversed after restoration of normal thyroid function [3].

\section{THE EFFECT OF L-THYROXINE SUPPLEMEN- TATION ON ARTERIAL STIFFNESS}

The major clinical question arising from the plethora of data that correlate hypothyroidism to an increase in CVD mortality through an increase in AS, is whether L-thyroxine supplementation reverses the aforementioned deleterious effects on functional vascular profile. This requires a balanced account of the strengths, weaknesses, and interpretation of the available literature in an unbiased fashion. The extent to which the observed associations are likely to be causal largely depends on a measurable improvement in objective parameters reflecting AS after restoration of euthyroidism. On that basis, methods of AS assessment vary among available studies. In a small older study [20], where A-mode ultrasonic device and B-M mode echo-tracking device were used for evaluation of carotid artery and radial artery diameter and wall-thickness treatment of hypothyroidism markedly reduced radial artery wall thickness $(-36 \%)$ and compliance $(-20 \%)$, although no changes in carotid artery function were observed. Of note, hypothyroid patients manifested overt disease with a median TSH of $80.6 \pm 23.5$ $\mu \mathrm{UI} / \mathrm{ml}$, whereas A- and B-M mode echo cannot be consid- ered as the gold standard method of functional vascular profile assessment [20]. These data however, constituted the primary basis for consecutive studies examining the potential beneficial effects of L-thyroxine supplementation.

The hemodynamic profile before and after levothyroxine treatment was evaluated in a group of 16 women with SubHypo (mean serum TSH: $17.1 \mu \mathrm{UI} / \mathrm{ml}$ ) and 15 women with overt hypothyroidism [47]. As expected, pre-treatment thyroid function was correlated to cardiac outpout $(\mathrm{CO})$ and SVR, whereas restoration of thyroid function resulted in 13$20 \%$ decrease in SVR in both groups. These favorable hemodynamic changes were also observed in a another small study (30 females with $\mathrm{SH}$ and 20 matched controls) where normalization of SVR assessed by cardiac magnetic resonance was evident after initiation of replacement therapy [48]. The question regarding the correlation between hypothyroidism and aortic stiffness (AoS) before and after thyroid replacement therapy was also investigated in a small Greek study, included patients with normal BP and hypothyroidism $(n=30)$, normotensive and hypothyroid subjects $(n=15)$ and also hypertensive and euthyroid patients $(n=15)$. AoS was calculated by measuring aortic systolic and diastolic diameter using a 2-dimensional M-mode ultrasound tracing. In both groups L-thyroxine replacement therapy was accompanied by a marked decrease in AoS and SVR. However, in patients with hypertension and hypothyroidism antihypertensive treatment with felodipine additively improved aortic elastic properties similarly to hypertensive and euthyroid patients. The main message of this study, besides the demonstrated beneficial effects of thyroxine supplementation, is that patients with hypertension and hypothyroidism have increased AoS and that thyroxine replacement therapy decreases AoS in all patiets and hypertension in $\sim 50 \%$ of them [49].

C-IMT has been also established as a significant parameter of arterial stiffening and thickening. The high reproducibility of this non-invasive ultrasonography method has been used to explore the efffects of hypothyroidism and Lthyroxine replacement on arterial stiffening. On that basis, 2 independent groups $[50,51]$ studied the effects of overt [50] and SubHypo [51] before and after supplementation therapy, focusing on potential changes in c-IMT $[50,51]$ and AS $\beta$ index [50]. In the first study (which included 45 patients with a mean $\mathrm{TSH}$ of $6.31 \mu \mathrm{UI} / \mathrm{ml})$ restoration of euthyroidism after 6 months resulted in a significant improvement in lipid profile and a reduction of mean c-IMT by $11 \%$. Although the beneficial effects of L-thyroxine replacement on c-IMT could be considered mild, this study [50] provided significant data regarding the key mechanisms involved in hypothyroidism-induced vascular alterations, implicating the improvement in lipid profile as a major determinant of this process. In the other study, the authors [51] also reported similar results regarding c-IMT decrease before and after treatment. In addition a marked improvement in AS $\beta$ index was demonstrated one year after restoration of euthyroidism. Baseline AS $\beta$ index levels and age correlated negatively with the observed changes in the vascular profile. The same group extended their data [24], by exploring the potential associations of the previous observed changes in c-IMT with serum levels of CRP as a useful predictor in AS improvement in hypothyroid patients. Similarly to previous findings 
[51] AS- $\beta$ levels decreased after L-thyroxine therapy, whereas these changes were negatively correlated with baseline levels of CRP. It becomes evident that available evidence differs in terms of the methods of assessment and interpretation of the findings regarding the beneficial effects of L-thyroxine replacement therapy. Therefore a useful approach to current data should consider their allocation according to their method of measurement. Available studies on this field $[23,52,53]$ are small, but all demonstrate a favourable effect of L-thyroxine supplementation on AS indexes, including AI, AG of central arterial pressure, reflected wave (TR) [23, 52] and PWV by measuring baPWV [53, 54]. Decreases in AI ranged from $28 \%$ [23] to $36.2 \%$ [52] after L-thyroxine treatment, whereas AG demonstrated significant reduction, in contrast to TR [23]. A marked improvement in baPWV values was also observed after restoration of euthyroidism $(1531.2$ vs $1330.2 \mathrm{~cm} / \mathrm{sec}, \mathrm{p}<0.005$, [53] and 1776.7 vs $1674.3 \mathrm{~cm} / \mathrm{sec}, \mathrm{p}=0.006$, [54]). These effects were observed in patients with profound hypothyroidism and SubHypo and were not accompanied by significant changes in the lipid profile or CRP in treated patients [52]. These results were also confirmed in a small well-designed study, where 19 female patients with SubHypo and 10 healthy controls were studied [55]. Assessment of arterial and cardiac function included pulse wave analysis and tissue Doppler dobutamine stress echocardiography. Baseline AG was significantly reduced after euthyroidism was achieved $(8.8 \pm 5.3 \mathrm{mmHg}$ ). TR also increased (from $139.3 \pm 11.7 \mathrm{~m} / \mathrm{sec}$ to $144.9 \pm 11.9 \mathrm{msec}$ ). No differences in cardiac function parameters were seen after L-thyroxine treatment [55].

Overall, our current view on the effects of L-thyroxine supplementation in overt and SubHypo on the vascular functional profile lies on the ground of small sample studies, with a wide heterogeneity in methods of assessing cardiovascular profile as well as in the definition of SubHypo and in the severity of overt hypothyroidism [3]. However, the vast majority, if not all, of the available evidence indicates a favourable effect of L-thyroxine on AS beginning in the very first few months of euthyroidism restoration. Moreover, data regarding the role of lipid profile and CRP levels in this process are conflicting. Until larger interventional studies are available, in the daily clinical setting, treatment initiation focusing on a reduction on CVD risk, particularly in SubHypo patients, should be tailored for each patient, according to the individual $\mathrm{CV}$ risk factors and demographic profile.

\section{THE EFFECT OF RESTORATION OF HYPER- THYROIDISM ON ARTERIAL STIFFNESS}

It is not clear whether or not CVD risk completely reverses with restoration of normal thyroid function in patients with hyperthyroidism. Few studies exist evaluating this topic and data are considered insufficient, due to both the small duration of observation and to the likelihood of developing hypothyroidism in the post-treatment period. In a small study in 27 patients with GD, which evaluated AS $\beta$ index (defined as the logarithm of the ratio of SBP to DBP divided by the fractional diameter increase during the cardiac cycle) in the common carotid artery, found that antithyroid drug treatment led to a significant decrease in AS $\beta$ index. This decrease correlated significantly with reductions in pulse pressure and retinal blood flow [39]. In a well-designed study of 20 thyro- toxic patients (19 with GD), central aortic AS was reassessed at 6 months after radioiodine treatment and in a euthyroid state. Surprisingly, the authors reported a rise in $\mathrm{AI}$ and AG post-treatment. Thyrotoxic patients demonstrated decreased AG of central pressure and reduced aortic AS compared with euthyroid controls. The results of this study were attributed by the authors to a cardio-protective effect of the lower AS, counteracting the effect of the increased in pulse pressure seen in thyrotoxicosis. Moreover, they suggested that age might also have played a role (mean age of participants $48.0 \pm 2.7$ years), proposing that their results might have been different in an elderly population [37].

To the best of our knowledge no study exists regarding the effect of thyroid function normalization in SubHyper patients on AS parameters, although it seems to be beneficial in other CVD indexes, such as reduction in heart rate, atrial and ventricular arrhythmias and left ventricular mass index either after methimazole treatment [56] or after radioiodine administration [57].

\section{CONCLUSION}

There is evidence that both hypothyroidism and hyperthyroidism are associated with increased AS, an emerging factor for increased CVD risk. However, the data are derived from small studies, in different populations and by using various methods for assessing AS. The restoration of thyroid function seems to reverse AS in hypothyroidism, although data are still limited. Therefore, we conclude that large multicenter randomized controlled trials are needed to both further elucidate the association of thyroid dysfunction with alterations of the arterial wall and to provide further evidence for the benefits of restoration of normal thyroid function on CVD risk

\section{CONFLICT OF INTEREST}

The author(s) confirm that this article content has no conflicts of interest.

\section{ACKNOWLEDGEMENT}

This review was written independently. The authors did not receive any funding for the preparation of the manuscript. The authors have given talks, attended conferences and participated in advisory boards and trials sponsored by various pharmaceutical companies.

\section{REFERENCES}

[1] Dörr M, Völzke H. Cardiovascular morbidity and mortality in thyroid dysfunction. Minerva Endocrinol 2005; 30: 199-216.

[2] Tseng FY, Lin WY, Lin CC, et al. Subclinical hypothyroidism is associated with increased risk for all-cause and cardiovascular mortality in adults. J Am Coll Cardiol 2012; 60: 730-7.

[3] Biondi B, Cooper DS. The clinical significance of subclinical thyroid dysfunction. Endocr Rev 2008; 29: 76-131.

[4] Ochs N, Auer R, Bauer DC, et al. Meta-analysis: subclinical thyroid dysfunction and the risk for coronary heart disease and mortality. Ann Intern Med 2008; 148: 832-45.

[5] Nanchen D, Gussekloo J, Westendorp RG, et al. PROSPER Group. Subclinical thyroid dysfunction and the risk of heart failure in older persons at high cardiovascular risk. J Clin Endocrinol Metab 2012; 97: 852-61

[6] Westerink J, van der Graaf Y, Faber DR, Spiering W, Visseren FL. SMART study group. Relation between thyroid-stimulating hor- 
mone and the occurrence of cardiovascular events and mortality in patients with manifest vascular diseases. Eur J Prev Cardiol 2012; 19: 864-73.

[7] Biondi B. Hypothyroidism as a risk factor for cardiovascular disease. Endocrine 2004; 24: 1-13.

[8] Anagnostis P, Karagiannis A, Tziomalos K, Athyros VG, Kita M, Mikhailidis DP. Endocrine hypertension: diagnosis and management of a complex clinical entity. Curr Vasc Pharmacol 2010;8: 646-60.

[9] Sawin CT, Geller A, Wolf PA, et al. Low serum thyrotropin concentrations as a risk factor for atrial fibrillation in older persons. $\mathrm{N}$ Engl J Med 1994; 331: 1249-52

[10] Sernia C, Marchant C, Brown L, Hoey A. Cardiac angiotensin receptors in experimental hyperthyroidism in dogs. Cardiovasc Res 1993; 27: 423-8.

[11] Völzke H, Robinson DM, Schminke U, et al. Thyroid function and carotid wall thickness. J Clin Endocrinol Metab 2004; 89: 2145-9.

[12] Klein I, Ojamaa K. Thyroid hormone and the cardiovascular system. N Engl J Med 2001; 344: 501-9.

[13] Davis P J, Davis FB. Nongenomic actions of thyroid hormone on the heart. Thyroid 2002; 12: 459-66.

[14] Laurent S, Cockcroft J, Van Bortel L, et al. European Network for Non-invasive Investigation of Large Arteries. Expert consensus document on arterial stiffness: methodological issues and clinical applications. Eur Heart J 2006; 27: 2588-605.

[15] Cavalcante JL, Lima JA, Redheuil A, Al-Mallah MH. Aortic stiffness: current understanding and future directions. J Am Coll Cardiol 2011; 57: 1511-22.

[16] Lehmann ED, Parker JR, Hopkins KD, Taylor MG, Gosling RG. Validation and reproducibility of pressure-corrected aortic distensibility measurements using pulse-wave-velocity Doppler ultrasound. J Biomed Eng 1993; 15: 221-8.

[17] Franklin SS, Khan SA, Wong ND, Larson MG, Levy D. Is pulse pressure useful in predicting risk for coronary heart Disease? The Framingham heart study. Circulation 1999; 100: 354-60.

[18] Laurent S, Boutouyrie P. Arterial stiffness: a new surrogate end point for cardiovascular disease? J Nephrol 2007; 20 (Suppl 12): S45-50.

[19] Vlachopoulos C, Aznaouridis K, Stefanadis C. Prediction of cardiovascular events and all-cause mortality with arterial stiffness: a systematic review and meta-analysis. J Am Coll Cardiol 2010; 55: 1318-27.

[20] Giannattasio C, Rivolta MR, Failla M, Mangoni AA, Stella ML, Mancia G. Large and medium sized artery abnormalities in untreated and treated hypothyroidism. Eur Heart J 1997; 18: 1492-8.

[21] Dagre AG, Lekakis JP, Papaioannou TG, et al. Arterial stiffness is increased in subjects with hypothyroidism. Int J Cardiol 2005; 103 : $1-6$.

[22] Lambrinoudaki I, Armeni E, Rizos D, et al. High normal thyroidstimulating hormone is associated with arterial stiffness in healthy postmenopausal women. J Hypertens 2012; 30: 592-9.

[23] Obuobie K, Smith J, Evans LM, John R, Davies JS, Lazarus JH. Increased central arterial stiffness in hypothyroidism. J Clin Endocrinol Metab 2002; 87: 4662-6.

[24] Nagasaki T, Inaba M, Shirakawa K, et al. Increased levels of Creactive protein in hypothyroid patients and its correlation with arterial stiffness in the common carotid artery. Biomed Pharmacother 2007; 61: 167-72.

[25] Lim VS. Thyroid function in patients with chronic renal failure. Am J Kidney Dis 2001; 38: 80-4.

[26] Blacher J, Pannier B, Guerin AP, Marchais SJ, Safar ME, London GM. Carotid arterial stiffness as a predictor of cardiovascular and allcause mortality in end-stage renal disease. Hypertension 1998; 32: $570-4$.

[27] Tatar E, Kircelli F, Asci G, et al. Associations of triiodothyronine levels with carotid atherosclerosis and arterial stiffness in hemodialysis patients. Clin J Am Soc Nephrol 2011; 6: 2240-6.

[28] Tatar E, Sezis DM, Kircelli F, et al. The association between thyroid hormones and arterial stiffness in peritoneal dialysis patients. Int Urol Nephrol 2012; 44: 601-6.

[29] Ojamaa K, Klemperer JD, Klein I. Acute effects of thyroid hormone on vascular smooth muscle. Thyroid 1996; 6: 505-12.

[30] Tognini S, Marchini F, Dardano A, et al. Non-thyroidal illness syndrome and short-term survival in a hospitalised older population. Age Ageing 2010; 39: 46-50.
[31] Meuwese CL, Carrero JJ, Cabezas-Rodríguez I, et al. Nonthyroidal illness: a risk factor for coronary calcification and arterial stiffness in patients undergoing peritoneal dialysis? J Intern Med 2013 Jul 1 [Epub ahead of print]

[32] Owen PJ, Chatterjee VK, John R, Halsall D, Lazarus JH. Augmentation index in resistance to thyroid hormone (RTH). Clin Endocrinol (Oxf) 2009; 70: 650-4

[33] Sellitti DF, Dennison D, Akamizu T, Doi SQ, Kohn LD, Koshiyama $H$. Thyrotropin regulation of cyclic adenosine monophosphate production in human coronary artery smooth muscle cells. Thyroid 2000; 10: 219-25.

[34] Bodlaj G, Pichler R, Brandstätter W, et al. Hyperthyroidism affects arterial stiffness, plasma NT-pro-B-type natriuretic peptide levels, and subendocardial perfusion in patients with Graves' disease. Ann Med 2007; 39: 608-16.

[35] Gu LQ, Zhao L, Zhu W, et al. Relationships between serum levels of thyroid hormones and serum concentrations of asymmetric dimethylarginine (ADMA) and N-terminal-pro-B-type natriuretic peptide (NT-proBNP) in patients with Graves' disease. Endocrine 2011 39: 266-71.

[36] Palmieri EA, Fazio S, Palmieri V, Lombardi G, Biondi B. Myocardial contractility and total arterial stiffness in patients with overt hyperthyroidism: acute effects of beta1-adrenergic blockade. Eur J Endocrinol 2004; 150: 757-62.

[37] Obuobie K, Smith J, John R, Davies JS, Lazarus JH. The effects of thyrotoxicosis and its treatment on central arterial stiffness. Eur J Endocrinol 2002; 147: 35-40.

[38] Czarkowski M, Hilgertner L, Powalowski T, Radomski D. The stiffness of the common carotid artery in patients with Graves' disease. Int Angiol 2002; 21: 152-7.

[39] Inaba M, Henmi Y, Kumeda Y, et al. Increased stiffness in common carotid artery in hyperthyroid Graves' disease patients. Biomed Pharmacother 2002; 56: 241-6.

[40] Akcay M, Akcay E, Yeter E, et al. Aortic elastic properties are impaired in patients with Graves' ophtalmopathy. Vasa 2011; 40: 41-8.

[41] Moulakakis KG, Sokolis DP, Perrea DN, et al. The mechanical performance and histomorphological structure of the descending aorta in hyperthyroidism. Angiology 2007; 58: 343-52.

[42] Gullu S, Altuntas F, Dincer I, Erol C, Kamel N. Effects of TSHsuppressive therapy on cardiac morphology and function: beneficial effects of the addition of beta-blockade on diastolic dysfunction. Eur J Endocrinol 2004; 150: 655-61.

[43] Tian L, Gao C, Liu J, Zhang X. Increased carotid arterial stiffness in subclinical hypothyroidism. Eur J Intern Med 2010; 21: 560-3.

[44] Owen PJD, Rajiv C, Vinereanu D, Mathew T, Fraser AG, Lazarus JH. Subclinical hypothyroidism, arterial stiffness and myocardial reserve. J Clin Endocrinol Metab 2006; 9: 2126-32.

[45] Imanishi R, Seto S, Toda G, et al. High brachial-ankle pulse wave velocity is an independent predictor of the presence of coronary artery disease in men. Hypertens Res 2004; 27: 71-8.

[46] Nagasaki T, Inaba M, Kumeda Y, et al. Increased pulse wave velocity in subclinical hypothyroidism. J Clin Endocrinol Metab 2006; 91: 154-8.

[47] Faber J, Petersen L, Wiinberg N, Schifter S, Mehlsen J. Hemodynamic changes after levothyroxine treatment in subclinical hypothyroidism. Thyroid 2002; 12:319-24.

[48] Ripoli A, Pingitore A, Favilli B, et al. Does subclinical hypothyroidism affect cardiac pump performance? Evidence from a magnetic resonance imaging study. J Am Coll Cardiol 2005; 45: 43945.

[49] Dernellis J, Panaretou M. Effects of thyroid replacement therapy on arterial blood pressure in patients with hypertension and hypothyroidism. Am Heart J 2002; 143: 718-24.

[50] Nagasaki T, Inaba M, Kumeda Y, et al. Decrease of arterial stiffness at common carotid artery in hypothyroid patients by normalization of thyroid function. Biomed Pharmacother 2005; 59: 8-14.

[51] Monzani F, Caraccio N, Kozàkowà M, et al. Effect of levothyroxine replacement on lipid profile and intima-media thickness in subclinical hypothyroidism: a double-blind, placebo- controlled study. J Clin Endocrinol Metab 2004; 89: 2099-106.

[52] Peleg RK, Efrati S, Benbassat C, Fygenzo M, Golik A The effect of levothyroxine on arterial stiffness and lipid profile in patients with subclinical hypothyroidism. Thyroid 2008; 18: 82530 . 
[53] Hamano K, Inoue M. Increased risk for atherosclerosis estimated by pulse wave velocity in hypothyroidism and its reversal with appropriate thyroxine treatment. Endocr J 2005; 52: 95-101.

[54] Nagasaki T, Inaba M, Yamada S, et al. Decrease of brachial-ankle pulse wave velocity in female subclinical hypothyroid patients during normalization of thyroid function: adouble-blind, placebocontrolled study. Eur J Endocriinol 2009; 160: 409-15.

[55] Owen PJD, Rajiv C, Vinereanu D, Mathew T, Fraser AG, Lazarus JH. Subclinical hypothyroidism, arterial stiffness and myocardial reserve. J Clin Endocrinol Metab 2006; 9: 2126-32.
[56] Sgarbi JA, Villaca F, Garbeline B, Villar HE, Romaldini JH. The effects of early antithyroid therapy for endogenous subclinical hyperthyroidism on clinical and heart abnormalities. J Clin Endocrinol Metab 2003; 88: 1672-7.

[57] Faber J, Wiinberg N, Schifter S, Mehlsen J. Hemodynamic changes following treatment of subclinical and overt hyperthyroidism. Eur J Endocrinol 2001; 145: 391-6.

(C) Anagnostis et al.; Licensee Bentham Open.

This is an open access article licensed under the terms of the Creative Commons Attribution Non-Commercial License (http://creativecommons.org/licenses/by-nc/3.0/) which permits unrestricted, non-commercial use, distribution and reproduction in any medium, provided the work is properly cited. 